\title{
LA CORA DE TUDMIR. DE LA ANTIGUEDAD TARDÍA AL MUNDO ISLÁMICO. Poblamiento y cultura material. Sonia Gutiérrez Lloret
}

\author{
(Casa de Velázquez (Madrid), Instituto de Cultura Juan Gil-Albert (Diputación de Alicante).
}

Madrid, 1996, 27×21 cm. )

\begin{abstract}
En los últimos años, el notable desarrollo de la arqueología medieval está permitiendo disponer de forma acelerada de una gran cantidad de documentación sobre la cultura material y los grupos de población que ocupaban el territorio de Al-Andalus. Esta información empieza lentamente a incorporarse a las obras de síntesis, cambiando en unas ocasiones y matizando en otras, algunas de las teorizaciones hechas antes de disponer de ella. Dentro de esta línea uno de los periodos que han recibido especial atención por parte de los arqueólogos es el que corresponde al de los primeros siglos, etapa que cubre la transición entre el mundo romano-visigodo y el islámico, y que cada vez se rebela más compleja, tal y como genialmente había descrito P. Guichard (1976) basándose exclusivamente en las fuentes escritas.
\end{abstract}

Para él, en Al-Andalus habían coexistido inicialmente dos tipos de organización social, una "occidental" y otra "oriental", aunque el autor francés dedicó preferentemente sus trabajos a esta última, insistiendo en la organización tribal de arabes y beréberes, lo que explicaba el mecanismo por el que estos pudieron absorber y transformar a una población indígena, inicialmente mucho más numerosa, dando lugar a una sociedad profundamente diferente de las feudales contemporáneas. Dentro de su propuesta los beréberes ocupaban un lugar de enorme importancia, ya que consideraba, en base a las fuentes escritas y a la toponimia, que estos habían llegado a la península en gran número, manteniendo sus estructuras tribales, lo que les había permitido berberizar parte del territorio de al-Andalus, y oponerse al control del incipiente Estado que trataban de implantar los omeyas.

A lo largo de los años ochenta, una serie de trabajos arqueológicos en el ámbito de las provincias de Málaga y Almería, permitieron a $M$. Acién señalar la gran importancia de los grupos campesinos indígenas, así como la existencia de materiales cerámicos que empezaban a aparecer en el siglo IX y que sólo podían considerarse como producciones de grandes alfares dedicados preferentemente al abastecimiento urbano. Estos elementos, junto a una relectura de las fuentes escritas, le llevarían a establecer que, tras la invasión árabe, se produjo un complejo proceso de transición en el que deben distinguirse tres formaciones sociales: la que da lugar al nuevo Estado islámico, a la que pertenecen sectores islamizados muy diversos, de los que los más evidentes son los grupos urbanos, la tribal, en la que se enmarcan algunos grupos árabes, pero sobre todo beréberes, que responden a las caracterizaciones realizadas por P. Guichard; y la heredera del mundo hispano-visigodo, en la que se inscriben las poblaciones rurales indígenas (1984; 1989). A estas últimas, o mas bien al grupo dominante de las mismas, dedicó un análisis pormenorizado (1994), demostrando que gracias a la forma de la conquista árabe de la Península, mediante pactos con la aristocracia visigoda, se habían mantenido buena parte de las relaciones sociales existentes previamente. Las contradicciones entre todos estos grupos acabarían provocando la fitna de finales del siglo IX, teorización con la que P. Guichard ha manifestado básicamente su acuerdo (GUICHARD 1995). De esta forma, las diferencias de fondo entre las diversas posiciones, aunque en algunos aspectos profundas, permiten un debate sereno a la búsqueda de la necesaria síntesis.

Toda esta línea de investigación se posiciona claramente tanto frente a quienes defienden que la única transición posible desde el mundo romano era hacia el feudalismo, como frente a los que parecen tratar de resucitar las antiguas tesis invasionistas, que solían considerar que las poblaciones indígenas no aportaban nada cuando eran sometidas por la "raza superior", portadora de civilización y conocimientos.

En este ámbito de análisis sobre la formación de AlAndalus, y del papel que la arqueología juega en las investigaciones actuales, es en el que se inscribe el libro de Sonia Gutiérrez, dedicado al territorio de la Cora de Tudmir, que comprendía parte de las provincias de Alicante, Murcia y Albacete. La autora aborda a lo largo de un denso volumen de más de 450 páginas, un gran número de cuestiones, con la claridad, rigor y honestidad intelectual que la caracterizan. La obra consta de una introducción y dos partes, la primera dedicada a los materiales y la segunda a los asentamientos y su articulación en el territorio.

En la introducción plantea, enfocándolos desde diversos ángulos, la situación en que se encuentran temas como la problemática histórica del periodo; posibilidades de la arqueología como fuente histórica; capacidad de distinguir a través de la cerámica distintos grupos humanos..., los mismos problemas que se van a retomar posteriormente en el estudio propiamente dicho, analizándolos en el marco de la documentación arqueológica obtenida en su área de trabajo. Como consecuencia de esto último, la autora se adhiere sin reservas a las propuestas de $M$. Acién que ya hemos descrito más arriba, lo que adelanta en la introducción, consiguiendo así en la obra una claridad en la discusión de los problemas que no suele ser frecuente.

La primera parte, dividida en dos capítulos, se centra en el estudio de los elementos materiales aparecidos en los yacimientos analizados en la segunda parte; el primero está dedicado a la cerámica y el segundo al resto de 
objetos (vidrio, metales, etc). Este último, como la misma autora indica, es sólo un catálogo, pero que proporciona datos acerca de la variedad de objetos de que se disponía en la época estudiada.

El estudio de la cerámica es con mucho más interesante y constituye en gran medida el núcleo "duro" de la obra, por lo que nos centraremos en él. Enlazando con las observaciones al respecto que ella misma realiza en la Introducción, empieza por abordar el problema del significado de la cerámica y sus posibilidades explicativas, al informar sobre un amplio número de rasgos como tecnología (dedicando un amplio apartado a analizar el proceso de fabricación), costumbres, etc. Pero además de ello, por su abundancia y durabilidad, es el que de forma más completa puede fechar los contextos. Y en arqueología este es el problema de partida. Todo elemento yacimiento, construcción del tipo que sea, sistema hidráulico- tiene que ser fechado por los materiales contenidos o asociados al mismo, o por su posición en un complejo estratigráfico, si ello no es posible, la asignación a una época u otra se convierte en un asunto muy problemático, que acaba dependiendo de la fe, y esta no tiene nada que ver con la arqueología.

La autora, perfectamente consciente de ello, dedica unos párrafos cruciales a la discusión sobre la clasificación. Con brevedad y precisión recoge las diferencias entre las dos principales interpretaciones de lo que representa una tipología, y diferencia estas de lo que es una sistematización, optando por establecer una de estas últimas, lo que justifica en que su objetivo no es construir una clasificación de tipo universal, útil para cualquier zona de la Península, sino una seriación de ámbito regional, ya que insiste en la regionalización que se produce durante los primeros siglos de Al-Andalus, apuntando nítidamente a la idea de que muy posiblemente había importantes diferencias entre los grupos de población de unas zonas y otras. Por ello, una tipología generalista haría desaparecer esas diferencias estableciendo una falsa homogeneización. De esta forma prosigue la línea de identificación y definición de zonas, idea que hasta cierto punto ya estaba contenida en las formulaciones iniciales de los estudios clásicos de Rosselló (1978) o Bazzana (1979 y 1980), y que ella misma empezó a establecer para el periodo emiral en esta misma región (GUTIÉRREZ LLORET 1988), y que para el resto del Sur de la Península múltiples investigaciones han confirmado sobradamente (VV.AA. 1993).

La sistematización propuesta por Sonia Gutiérrez Lloret se estructura en dos niveles. El primero parte por un lado de las técnicas de fabricación (mano, torneta, torno) y por otro del tipo de acabado (vidriada y sin vidriar). Unos criterios y otros fueron establecidos a finales de los ochenta, habiendo tenido la autora una importante contribución en ello, y estando hoy plenamente aceptados por la totalidad de los especialistas que trabajan en este campo.

Para el segundo nivel, ha optado por establecer una clasificación compuesta por 34 series, definidas en base a criterios formales/ funcionales, cada una de las cuales consta de varias formas relacionadas entre sí por determinados atributos. Como ella misma admite, el conjun- to podría considerarse como una tipología, en la que las series serían los Tipos y las formas las Variantes, pero estamos de acuerdo con ella en que el notable número de series establecidos, y la proliferación de formas, impiden considerarlo así.

Es precisamente esta abundancia de series y formas lo que diferencia su propuesta de la mayoría de las existentes, y lo que la aleja de alguna de las más recientes, que precisamente apuestan por secuencias alfanuméricas con un número de series muy reducido (ACIÉN et alii. 1991). Pero su opción se corresponde perfectamente con su intención expresa de definir de la forma más completa posible el universo de elementos cerámicos presentes en su territorio de investigación, algo imprescindible para desarrollar análisis ulteriores. Porque efectivamente, de esta manera consigue organizar la cerámica de forma que pueda relacionar inmediatamente cada forma con nuevos materiales que puedan aparecer, con lo que la cronología de lo ya estudiado podrá ir ajustándose sin necesidad de nuevas revisiones exhaustivas.

No obstante, como la autora reconoce y asume, el método parece excesivamente subjetivo. Aunque cualquier sistema de clasificación lo es, puesto que es siempre quien realiza la clasificación el que define los rasgos a tener en cuenta, creo que en la actualidad la informática, a través de los análisis multivariantes de rasgos morfométricos, puede simplificar e incluso hasta cierto punto objetivizar, sistematizaciones de este tipo.

Pero la clasificación cerámica es sólo un medio para un fin, y este es, primero, el establecimiento de una cronología, y luego la identificación de los grupos humanos que fabricaron ese material, y todo ello como paso previo al análisis del poblamiento, y de las estructuras económicas y sociales que sea posible inferir del mismo.

Desde el punto de vista cronológico, para muchos investigadores no arqueólogos resulta descorazonadora la amplitud del arco cronológico que se atribuye a estas cerámicas, ya que los conjuntos suelen fecharse simplemente como emirales, lo que supone un arco temporal de dos siglos. Quizá sea preciso recordar que hace apenas veinte años toda la cerámica posterior a las últimas sigillatas se incluía en un cajón de sastre indiferenciado que abarcaba desde época islámica hasta el presente, y que sólo empezó a aclararse a partir de la sistematización de Rosselló (1978) y del trabajo de muchos otros investigadores. De hecho, sólo a finales de los años ochenta empezaron a establecerse series coherentes para la fase emiral, ingente tarea en la que precisamente Sonia Gutiérrez jugó un papel de primera importancia. En estos últimos diez años están definiéndose las series regionales. Si comparamos este avance con el siglo de investigaciones que, por ejemplo, acumula la cerámica de época ibérica, y que sólo muy recientemente está empezando a poder fecharse, y sólo en algunas zonas, por arcos cronológicos inferiores a cincuenta años, parece evidente que el avance en el estudio de la cerámica andalusí es espectacular.

Volviendo al análisis de Sonia Gutiérrez, la autora consigue establecer tres grupos, el primero con crono- 
logías de los siglos VI a VIII, otro con cronología específicamente emiral, de la $2^{\mathrm{a}}$ mitad del VIII al IX y el último centrado en el siglo $X$.

La cronología del primero la proporcionan una serie de importaciones norteafricanas, que aparecen junto a vasijas a mano y torneta. La importancia de esta parte reside precisamente en que la definición de las cerámicas anteriores a la invasión es un elemento fundamental para el estudio de las que aparecen en época emiral.

La segunda fase se va a distinguir por la desaparición de cualquier tipo de cerámica importada, o de las que pueden considerarse de "lujo", y el dominio absoluto de materiales a mano y torneta que parecen claramente derivados de los anteriores, lo que demuestra que las poblaciones que los utilizan son indígenas, así como por la aparición de otras formas, como los tananir y los arcaduces, que para la autora fueron introducidos por los conquistadores, junto al uso poco después del torno rápido y del vidriado. Estos dos últimos elementos se harán dominantes en la tercera fase, demostrando la generalización de los sistemas de producción casi "industriales", aunque desde luego con muchos centros productores, y sin llegar nunca a los niveles de concentración productiva que se dieron en el Bajo Imperio romano. Esta concentración de la producción estuvo muy posiblemente vinculada al desarrollo del nuevo urbanismo impulsado por la islamización -en el sentido expresado por M. Acién- de la sociedad.

Como la misma autora reconoce, el arco cronológico de cada una de estas producciones es aún excesivamente amplio, y por ello su utilidad resulta inevitablemente limitada. Pero la consecución de una mayor precisión sólo podrá obtenerse a partir de la realización de estudios regionales como el realizado, que sistematicen las producciones y permitan integrar en ellos los nuevos datos que las excavaciones con secuencias estratigráficas, de las que ahora, para todo el Sur y Este peninsular apenas disponemos de media docena

Por lo que se refiere a la cuestión de si la cerámica es capaz de dar cuenta realmente de los diversos grupos de población, las investigaciones de Sonia Gutiérrez en la Cora de Tudmir confirman las hipótesis de M. Acién acerca de la importancia de las poblaciones indígenas herederas del mundo hispano-visigodo, a las que pertenece la inmensa mayoría de la cerámica estudiada, y junto a la que aparecen algunos materiales, como los arcaduces del Bajo Segura, que presentan semejanzas con producciones egipcias actuales. $Y$ para finales del IX $y$ el $X$, la existencia de conjuntos cerámicos producidos por la nueva sociedad islámica urbana, mientras que no aparecen materiales que puedan asignarse a grupos beréberes.

A pesar de la extensión y densidad del estudio, muchas cuestiones quedan en la obra apenas esbozadas. Así por ejemplo, por lo que se refiere a los mencionados arcaduces, aunque la autora se limita a advertir ese dato, sin atribuirle por el momento otro valor, la realidad es que este tipo de elementos plantea el problema -o más bien la evidencia histórica- de la pervivencia de aspectos básicos de la cultura material de los grupos campesinos, cues- tión por ejemplo muy evidente en el uso hasta la actualidad de la tecnología empleada en los sistemas de regadio andalusíes. Estas cuestiones son un "problema" desde planteamientos ideológicos que consideran que la única historia existente es la del "desarrollo" y el "progreso", y que todo lo que no se ajuste a ese dogma es parálisis y retroceso. Es claro en esta perpsectiva que el estudio de las sociedades campesinas está aún por hacer, aunque desde distintas posiciones se intentan crear los instrumentos conceptuales necesarios para hacerla posible (SEVILLA y GONZÁLEZ eds. 1992).

Otra cuestión, más presente en la obra, es la de la falta de constatación de grupos bereberes. En realidad, la presencia de estos en el Sur y Sureste de Al-Andalus sigue resultando extremadamente opaca, como se puso de manifiesto en los debates sobre la cerámica emiral desarrollados en Salobreña en 1990 (V.AA. 1993), sin que las investigaciones posteriores hayan cambiado por el momento el panorama. Ello resulta paradójico porque precisamente una parte de los trabajos arqueológicos iniciales trataban de justificar las elaboraciones realizadas por P. Guichard a partir de las fuentes escritas. Ello nos llevó a muchos arqueólogos a cometer un error de bulto, esto es, tratar de identificar asentamientos beréberes sin haber comprobado previamente en el Norte de Africa cuales eran los materiales que pudieron traer consigo dichas poblaciones, al partir del apriorismo de que tenían que resultar fácilmente identificables (SALVATIERRA, CASTILLO 1992). Hoy está claro que el primer paso es conocer previamente la cerámica que esos grupos hipotéticamente trajeron o utilizaron.

Los datos seguros conocidos para esta época del Norte de Africa, muy escasos y poco sistematizados, son también recogidos por Sonia Gutiérrez Lloret, que señala su relativa semejanza con los peninsulares. Pero advierte que esos materiales proceden de antiguos núcleos romanizados, lo que indica que el mundo tardorromano de ambas orillas del Mediterráneo fue bastante homogéneo y debió evolucionar de forma similar, pero por el contrario nada se sabe del material que pudieron tener los grupos beréberes sin contacto con dichos núcleos. Por ello la autora, con exquisita prudencia, se detiene en este punto y se abstiene de hacer otras inferencias.

No obstante, a nivel de hipótesis, puede plantearse que en realidad esas cerámicas a mano localizadas corresponden también en gran medida a los grupos beréberes de esas áreas, que por otra parte serían los mismos que entrarían en contacto con los árabes y que se trasladarían a la Península. De esta forma, los materiales traídos por esos grupos serían relativamente semejantes a los indígenas peninsulares. Y esta puede ser la causa de que aún nos resulten invisibles, ya que nuestro conocimiento de la cerámica no ha llegado todavía al estadio que nos permita diferenciarlos. La investigación que en la actualidad desarrollan en el marco de la cooperación franco marroquí investigadores de la Casa de Velázquez y del INSAP (Rabat) junto con algunos españoles en el actual Marruecos, permitirá la obtención de series más completas de las hoy existentes, contemporáneas de las emirales, y a partir de ello efectuar comparaciones con las de la Península. 
Ante esta situación, el establecimiento de series sistemáticas como la efectuada por Sonia Gutiérrez es el mejor camino para detectar las diferencias entre asentamientos y áreas, y las hipotéticas soluciones de continuidad con los materiales anteriores a la conquista, y avanzar así en la identificación tanto de los asentamientos indígenas, como de los no indígenas. Quizá puedan emplearse otros elementos arqueológicos para detectar la presencia de estos últimos, pero la única propuesta presentada hasta ahora, la de M. Barceló y sus colaboradores (1995), para quienes la huella de los beréberes son los sistemas de regadío, y que por tanto debe recurrirse a la arqueología hidráulica, choca con el problema de que no hay pruebas arqueológicas de que la ecuación beréberes= hidráulica sea correcta o excluya otras; mientras que por otra parte resulta casi imposible fechar dichos sistemas en sí mismos, ya que además de su esencial simplicidad en cuanto a los materiales empleados, han seguido construyéndose de forma similar hasta hace pocas décadas, como ya se ha indicado. El procedimiento de fecharlos mediante los asentamientos próximos, resulta como mínimo problemático, como sabe cualquier arqueó-logo con un mínimo de experiencia, a parte de que hasta el momento esos despoblados presentan cerámica de tipo indígena o son materiales que parecen relacionarse mejor con otros ámbitos, como las cerámicas producidas en grandes centros de principios del siglo $X$, o los arcaduces del Bajo Segura ya citados, de dificil adscripción.

Como se advierte, el problema de la localización, organización e interrelación de los grupos rurales existentes en el emirato ha resultado más complejo de lo que inicialmente se creía, y ello ha dado lugar a algunas reacciones bastante sorprendentes y totalmente fuera de lugar. La profundidad de la problemática que los análisis arqueológicos están poniendo de manifiesto demuestran la gran potencia de la arqueología, y la necesidad de aplicar en la investigación una metodología rigurosa. La arqueología no puede limitarse a corroborar las deducciones obtenidas a partir de la documentación escrita, sino que, realizados los análisis arqueológicos, deberá irse necesariamente a una nueva síntesis en la que estén todos los elementos. Esto, que es la base de la investigación histórica, parece ser olvidado en algunas ocasiones, ante la evidente dificultad de operar seriamente con documentación arqueológica, para lo que hace falta una preparación extremadamente sólida, que no se adquiere por el mero hecho de proclamar que se tiene.

Por todo esto, la opción de Sonia Gutiérrez de circunscribir su clasificación a la Cora de Tudmir, e indicar la imposibilidad de hacerla extensiva mecánicamente a otras zonas nos parece totalmente correcta. La cerámica de la zona muestra una inequívoca relación con los materiales existentes en la misma área antes de la invasión musulmana, por lo que considera la zona como de poblamiento casi exclusivamente indígena, lo que por otro lado se relaciona bien con lo que se conoce a través de la documentación escrita, y confirma el contenido fundamental -en lo que a población se refiere- del pacto de Teodomiro.

Después de la sistematización morfológica de la cerámica, la autora se extiende ampliamente sobre la asigna- ción funcional de cada serie, mostrando un exhaustivo conocimiento y una cuidada documentación, de cada una de ellas. Prosigue luego con el análisis de las decoraciones, que ofrecen variantes bastante limitadas y generalmente toscas, aunque hay algunas que presentan interesantes posibilidades de cara al establecimiento de paralelos y a la fijación cronológica.

La segunda parte del libro está dedicada al poblamiento y el territorio, dividida también en dos capítulos. La misma autora advierte que no es un estudio del poblamiento en sentido estricto, ya que falta un análisis sistemático del territorio.

El trabajo recoge casi medio centenar de asentamientos, investigados con distintos niveles de profundidad durante las últimas décadas por diversos estudiosos, y fechados en época emiral mediante los materiales estudiados en la primera parte, y en algunos casos también mediante la documentación escrita. Se trata de una sistematización hasta cierto punto similar a la realizada con la cerámica, contemplando seis series, además de edificaciones religiosas aisladas y cementerios. Dentro de esos asentamientos cabe distinguir dos grupos.

El primero son las ciudades, considerando como tales aquellos lugares que eran ciudades en época romana, las que son citadas como tales en el pacto de Teodomiro, y las denominadas madina en las fuentes árabes. De cada una de ellas se analizan los datos disponibles para la época estudiada, que en general son muy escasos, tratando de establecer el nivel en que se encontraban antes de la conquista, y su evolución posterior a esta. Así, la autora consigue determinar que algunas de ellas experimentaron un cierto renacimiento en época visigoda, quizá en el marco de las luchas con Bizancio, y que llegaron como núcleos de cierta entidad hasta el siglo IX, para decaer o incluso desaparecer después, mientras eran sustituidas por otras de nueva fundación. No se trata simplemente de una reiteración de los textos publicados con anterioridad, ya que en general se aportan nuevos datos, que resultan espectaculares en el caso del Tolmo de Minateda, la posible Iyi(h) mencionada en el Pacto de Teodomiro, actualmente en excavación.

En el segundo grupo se recogen los demás asentamientos. Con buen criterio la autora ha situado los datos básicos y la descripción de cada yacimiento, así como los materiales seleccionados de algunos de ellos, en un apéndice al final del volumen, pudiendo centrarse así en este capítulo en la problemática histórica general que presenta cada uno de los grupos establecidos (poblados de altura; asentamientos rurales en llano; en cabezos o laderas; poblados fortificados y castillos) y las particularidades que en ese aspecto ofrecen los de la zona estudiada. Una inteligente utilización de la bibliografía más reciente y de la documentación escrita existente para la zona, le permite avanzar para algunos de ellos hipótesis sugestivas, aunque la falta de excavaciones sistemáticas limita la concrección de las mismas, al tiempo que impide conocer la organización interna de cada uno de esos tipos de asentamiento, y profundizar en cuestiones de crucial impor- 
tancia como la organización social de la población de cada uno de ellos, temas que habrá que desarrollar en el futuro, posiblemente en la línea planteada por M. Acién (|99|).

El último capítulo está muy limitado por la falta de estudios específicos, dado lo reciente de las investigaciones en el campo de la arqueología del paisaje. A pesar de ello, aborda el reto de relacionar la problemática histórica concreta de la zona, con los asentamientos y las áreas productivas. Para ello retoma el problema de la discontinuidad urbana, señalando que como consecuencia de la decadencia de la ciudad romana, debió producirse el fortalecimiento de los grandes propietarios rurales, y la formación, como respuesta, de comunidades en los márgenes del territorio controlado por los primeros. La mencionada falta de estudios previos le impiden establecer por ahora cuales fueron los procesos económicos que llevaron al desarrollo de las nuevas ciudades islámicas, y como eran los sistemas productivos de las zonas centrales y más ricas del territorio, teniendo que conformarse con el análisis de los sistemas productivos creados por algunos de los grupos "marginales", centrándose en los ejemplos de El Zambo (poblado de altura) y de los cabezos situados en los marjales del Bajo Segura, lo que a pesar de todo es suficiente para dar una buena idea de la diversidad de respuestas de la población.

Para terminar, se refiere brevemente al problema de los caminos y vías de comunicación, un tema de gran importancia, pero que en la práctica apenas ha sido aún estudiado, fuera de generalizaciones como la de suponer la pervivencia de las vías romanas, lo que la autora indica que debe matizarse considerablemente.

En resumen, un libro de gran importancia, que marca indudablemente el camino a seguir. La profundización en el conocimiento de la historia social y económica de AlAndalus en general, y de su etapa emiral en particular, vendrá en gran medida de la multiplicación de textos como este, donde se analicen en profundidad y sistemáticamente las diferentes zonas del territorio, -tanto regionales como "locales" ( yacimientos sistemáticamente estudiados) y que permitan compararlas, determinando en cada una que grupos humanos se establecieron, su articulación, y la variedad de respuestas económico-sociales que dieron. Frente a las visiones que parecen considerar que la formación de Al-Andalus fue un proceso muy simple, de sustitución de una sociedad por otra, de unas formas económicas por otras, el estudio realizado por Sonia Gutiérrez, la multiplicidad de temas tocados y la variedad de ángulos de enfoque, indican la complejidad del mismo, y obligan, a estudios arqueológicos del máximo rigor, desechando por completo los apriorismos pseudocientíficos, y las verdades reveladas.

Queda por último referirse a la edición realizada por la Casa de Velázquez de Madrid y el Instituto de Cultura Juan Gil-Albert de la Diputación de Alicante. Se trata de un volumen de $27 \times 21 \mathrm{~cm}$. encuadernado en tela, y con una cuidadosa impresión, tanto de texto como de figuras, como es costumbre en la colección en que aparece. No cabe sino felicitar a ambas instituciones por haber abor- dado la edición de una obra de indudable importancia, tanto por sus aportaciones históricas, como por la metodología que de ella se deriva.

Vicente Salvatierra

\section{BIBLIOGRAFÍA}

ACIÉN, M. (1984): "La formación y destrucción de AlAndalus". Historia de los Pueblos de España. Tierras Fronterizas (I). Andalucía. Canarias. Barcelona.

ACIÉN, M. (1989): "Poblamiento y fortificación en el sur de Al-Andalus. La formación de un país de Husun". III Congreso de Arqueología Medieval Española (Oviedo 1989) Vol. I, Ponencias. Oviedo, pp. I35-I50.

ACIÉN, M. (|99|): "Recientes estudios sobre arqueología andalusí en el Sur de Al-Andalus". Aragón en la Edad Media vol. IX, Zaragoza, pp. 355-369.

ACIÉN, M. (1994): Entre el feudalismo y el islam. "Umar ibn Hafsun en los historiadores, en las fuentes y en la historia. Jaén. (2ª Ed. ampliada 1997)

ACIÉN, M.; et alii. (!99I): "Evolución de los tipos cerámicos en el SE de Al-Andalus" V Colloque International sur la céramique médievale en Méditerranée Occidentale. Rabat. (Tirada a parte).

BARCELÓ, M.; et al. (1995): El agua que no dueme. Granada

BAZZANA, A. (1979): "Céramiques médiévales: les methodes de la decription analytique appliquées aux productions de l'Espagne orientale." Mélanges de la Casa de Velázquez, XV, pp. I35-185.

BAZZANA, A. (1980): "Céramiques médiévales: les methodes de la decription analytique appliquées aux productions de l'Espagne orientale. II. Les poteries décorées. Chronologie des productions médiévales". Mélanges de la Casa de Velázquez, XVI, pp. 57-95.

GUICHARD, P. (1976): Al-Andalus. Estructura antropológica de una sociedad islámica en occidente. Barcelona.

GUICHARD, P. (1995): La España musulmana. Al-Andalus omeya (Siglos VIII-XII). Historia 16, vol. 7 Madrid.

GUTIÉRREZ LLORET, S. (1988): Cerámica común paleoandalusí del Sur de Alicante (Siglos VII-X). Alicante. ROSSELLÓ, G. (1978): Ensayo de sistematización de la cerámica árabe de Mallorca. Palma de Mallorca.

SALVATIERRA, V:; CASTILLO, JC. (I992): "El Cerro de Peñaflor. Un posible asentamiento beréber en la campiña de Jaén". Anaquel de Estudios Arabes, III; pp. |53-I6I.

SEVILLA, E; y GONZÁLEZ, M. (Eds.) (1992): Ecología, campesinado e historia. Madrid.

W.AA. (1993): La cerámica altomedieval del Sur de Al-Andalus. (Salobreña 1990). Granada 